\title{
Long noncoding RNA SNHG12 promotes tumour progression and sunitinib resistance by upregulating CDCA3 in renal cell carcinoma
}

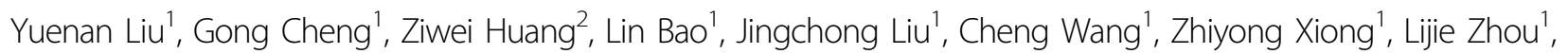
Tianbo Xu', Di Liu', Hongmei Yang $\mathbb{1}^{3}$, Ke Chen' and Xiaoping Zhang ${ }^{1}$

\begin{abstract}
Renal cell carcinoma (RCC) is one of the most frequently observed malignant tumours in the urinary system and targeted drug resistance is quite common in RCC. Long noncoding RNA SNHG12 (IncRNA SNHG12) has emerged as a key molecule in numerous human cancers, but its functions in renal cell carcinoma (RCC) sunitinib resistance remain unclear. In this study, we found SNHG12 was highly expressed in RCC tissues and in sunitinib-resistant RCC cells and was associated with a poor clinical prognosis. SNHG12 promoted RCC proliferation, migration, invasion and sunitinib resistance via CDCA3 in vitro. Mechanically, SNHG12 bound to SP1 and prevented the ubiquitylation-dependent proteolysis of SP1. Stabilised SP1 bound to a specific region in the promoter of CDCA3 and increased CDCA3 expression. Furthermore, in vivo experiments showed that SNHG12 increased tumour growth and that knocking down SNHG12 could reverse RCC sunitinib resistance. Our study revealed that the InCRNA SNHG12/SP1/CDCA3 axis promoted RCC progression and sunitinib resistance, which could provide a new therapeutic target for sunitinibresistant RCC.
\end{abstract}

\section{Introduction}

Renal cell carcinoma (RCC) is one of the most frequently observed malignant tumours in the urinary sys$\mathrm{tem}^{1}$. Among the various pathological types of RCC, $\sim 70-80 \%$ are clear cell renal cell carcinoma $(\mathrm{ccRCC})^{2}$. It is estimated that the number of new cases of RCC will be 73,820 , and a total of 14,770 mortalities will be reached in the USA in $2019^{3}$. The diagnosis of RCC at an early stage is not easy because the initial symptoms are usually not obvious. In fact, for $\sim 30 \%$ of RCC patients, cancer has

\footnotetext{
Correspondence: Ke Chen (shenke@hust.edu.cn) or

Xiaoping Zhang (xzhang@hust.edu.cn)

'Department of Urology, Union Hospital, Tongji Medical College, Huazhong

University of Science and Technology, No. 1277 Jiefang Avenue, 430022

Wuhan, China

${ }^{2}$ Department of Breast and Thyroid Surgery, Union Hospital, Tongji Medical College, Huazhong University of Science and Technology, No. 1277 Jiefang Avenue, 430022 Wuhan, China

Full list of author information is available at the end of the article

These authors contributed equally: Yuenan Liu, Gong Cheng, Ziwei Huang

Edited by G. Calin
}

already metastasised at first diagnosis ${ }^{4}$. Although targeted drugs, such as sunitinib, have been used to treat advanced RCC patients, unfortunately, drug resistance develops within 6-15 months, and the overall survival remains unsatisfactory $^{5-7}$. As a result, it is necessary to elucidate the potential mechanisms underlying RCC progression and drug resistance.

Long noncoding RNAs (lncRNAs) are defined as a group of RNAs with a minimum length of 200 nucleotides that do not encode proteins ${ }^{8}$. However, studies have demonstrated their significant roles in the regulation of transcription and translation of protein-coding genes ${ }^{9-11}$. Based on these functions, lncRNAs are involved in multiple oncogenic processes, including tumour cell proliferation, metastasis, apoptosis inhibition and drug resistance $^{12-14}$. Small nucleolar RNA host gene 12 (SNHG12) has been increasingly reported to participate in diverse cancers ${ }^{15}$. It is believed that SNHG12 could act as a molecular sponge of microRNAs to promote tumour

\section{(c) The Author(s) 2020}

(c) (i) Open Access This article is licensed under a Creative Commons Attribution 4.0 International License, which permits use, sharing, adaptation, distribution and reproduction c. in any medium or format, as long as you give appropriate credit to the original author(s) and the source, provide a link to the Creative Commons license, and indicate if changes were made. The images or other third party material in this article are included in the article's Creative Commons license, unless indicated otherwise in a credit line to the material. If material is not included in the article's Creative Commons license and your intended use is not permitted by statutory regulation or exceeds the permitted use, you will need to obtain permission directly from the copyright holder. To view a copy of this license, visit http://creativecommons.org/licenses/by/4.0/. 
proliferation, metastasis and epithelial-mesenchymal transition ${ }^{16,17}$. Furthermore, SNHG12 is also involved in multidrug resistance ${ }^{18,19}$. However, the functions and mechanisms of SNHG12 in RCC remain unclear.

In the present study, we screened lncRNAs involved in sunitinib-resistant RCC by bioinformatics analysis. We found that lncRNA SNHG12 was highly expressed and could be a diagnostic and prognostic biomarker of RCC. In addition, overexpression of SNHG12 promoted RCC progression and sunitinib resistance both in vitro and in vivo. By further exploration, we determined that SNHG12 could upregulate CDCA3 by stabilising the transcription factor SP1. Therefore, our study describes a novel SNHG12/SP1/CDCA3 axis that might be a promising therapeutic target for RCC.

\section{Results}

\section{SNHG12 was overexpressed and indicated a poor clinical} prognosis in RCC

Accumulating studies have demonstrated that lncRNAs play important roles in tumour progression and drug resistance. We sequenced 3 pairs of RCC and adjacent normal tissues and analysed the differential expression of lncRNAs. We also studied the differential expression of lncRNAs between tumour and normal tissues from the TCGA-KIRC database. Moreover, differentially expressed lncRNAs between sunitinib-resistant RCC and sunitinibsensitive RCC were determined from the GEO database $\left(\right.$ GSE64052 $\left.2^{20}\right)$. From the intersection of these three datasets, 4 lncRNAs were obtained, two of which were upregulated and two were downregulated (Fig. 1a, b). For further analysis, we mined data in the TCGA-KIRC database and in Yusenko Renal ${ }^{21}$ from the Oncomine database. Among the four identified lncRNAs, only the SNHG12 expression level was correlated with the overall survival time, tumour grade and tumour stage of ccRCC patients (Fig. 1c, d and Supplementary Fig. 1). Higher SNHG12 expression indicated shorter survival time and higher tumour grade and stage (Table 1). Univariate and multivariate analyses were performed demonstrating that SNHG12 was one of the independent prognostic markers of ccRCC (Table 2). SNHG12 was expressed at a higher level in tumour tissues than in normal tissues, and the receiver operating characteristic (ROC) curve showed that SNHG12 could be used as a diagnostic biomarker for ccRCC (Fig. 1e).

To verify the above results, RCC cell lines and more tumour tissues were added to the study. As shown in Fig. 1f, SNHG12 expression levels were significantly higher in RCC cells and tissues. According to the cellular fractionation assay of RNA, SNHG12 was mainly located in the nucleus in RCC cells (Fig. 1g). Furthermore, RNA fluorescence in situ hybridisation assays also produced the same results (Fig. $1 \mathrm{~h}$ and Supplementary Fig. 2).
Therefore, we found that nucleus-located lncRNA SNHG12 was highly expressed in RCC and was a predictor of a poor clinical prognosis.

\section{SNHG12 increased the proliferation, invasion and migration of RCC cells in vitro}

To determine the biological function of SNHG12 in RCC cells, we transfected short hairpin RNA (shRNA) or the pcDNA3.1 vector to knockdown or overexpress SNHG12 in 786-O and ACHN cells. Quantitative reverse transcription PCR (qRT-PCR) was used to determine the effect of transfection (Fig. 2a). CCK8 assays showed that knocking down SNHG12 expression significantly reduced the proliferation ability of 786-O cells and ACHN cells; conversely, overexpressing SNHG12 increased cell proliferation (Fig. 2b). Transwell assays were used to assess the migration and invasion ability of cells. SNHG12 knockdown resulted in a lower 786-O cell and ACHN cell migration and invasion rate, while SNHG12 overexpression showed the opposite results (Fig. 2c, d). In addition, cell cycle assays suggested that knockdown of SNHG12 inhibited cell cycle progression from G0/G1 phase to $S$ phase. More cells were arrested at G0/G1 phase, so cell proliferation decreased. In contrast, overexpression of SNHG12 promoted entry of more cells into $\mathrm{S}$ phase so that the cell proliferation rate increased (Fig. 2e, f). In summary, SNHG12 increased the proliferation, invasion and migration of RCC cells.

\section{SNHG12 upregulated the expression of CDCA3 in RCC cells}

To investigate the potential mechanism of SNHG12 promoting the progression of RCC, GSEA analysis was performed based on data from GEO database $\left(\right.$ GSE $\left.53757^{22}\right)$. As shown in Supplementary Fig. 3a-f, high expression of SNHG12 was related to many signalling pathways that are clearly involved in tumourigenesis and metastasis, such as the cell cycle checkpoint, cell migration, drug resistance, and epithelial-mesenchymal transition. We also accessed top 100 genes that related with SNHG12 by GSEA analysis, which was shown in the heatmap (Fig. 3a). With the intersection of these two parts, 6 tumour-related genes were selected (Supplementary Table 1). Then, the mRNA expression level of the 6 genes was detected by qRT-PCR after knocking down or overexpressing SNHG12 in 786-O or ACHN cells. Analysis of these six genes demonstrated that only the CDCA3 mRNA expression level changed similar to SNHG12 (Fig. 3b and Supplementary Fig. 3g, h). Western blotting showed that at the protein level, SNHG12 could regulate CDCA3 expression (Fig. 3c). In other words, the CDCA3 expression level might be positively regulated by SNHG12.

CDCA3, cell division cycle associated 3 , has been reported to participate in numerous tumours, including 


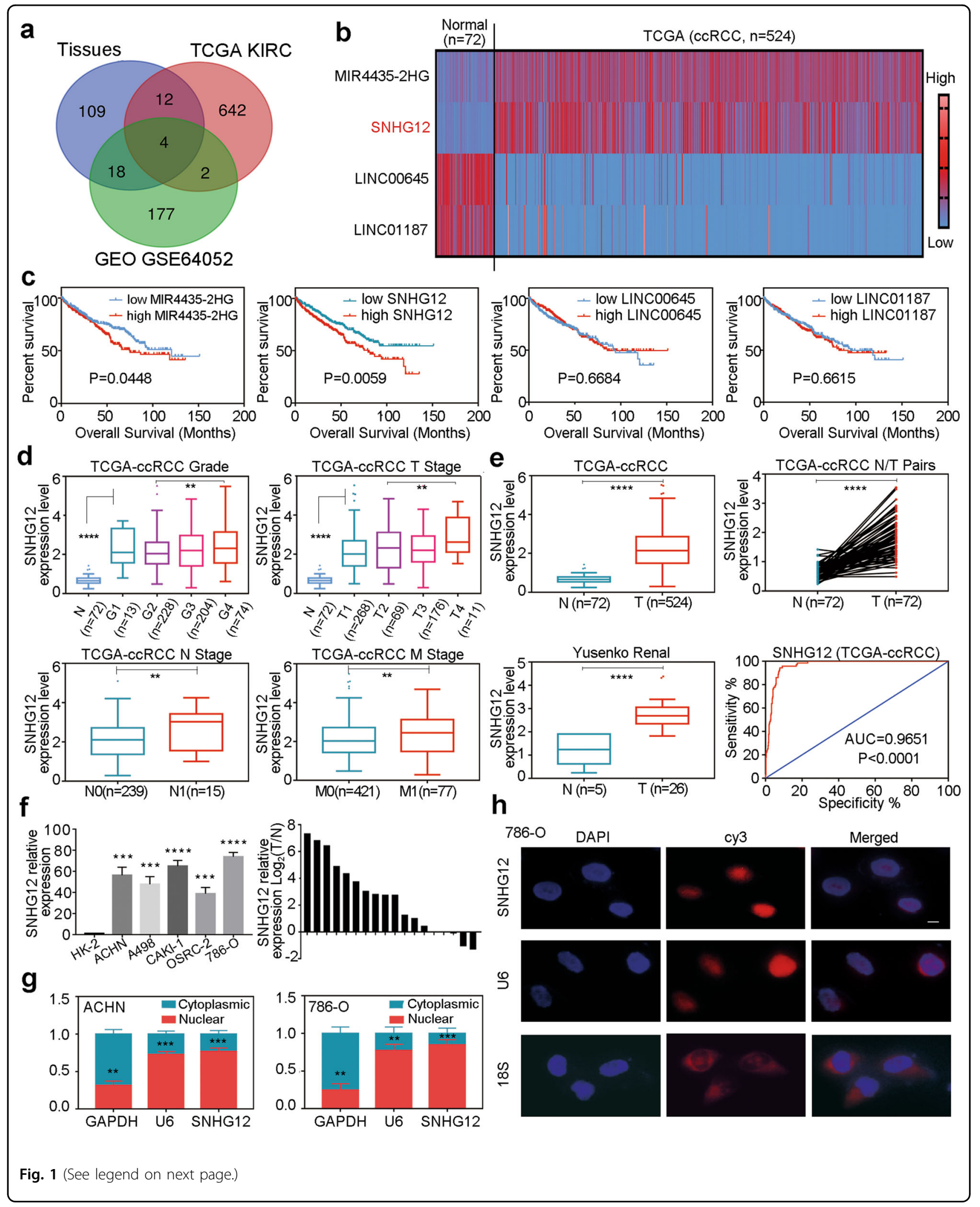


(see figure on previous page)

Fig. 1 SNHG12 was overexpressed and indicated a poor clinical prognosis in RCC. a A Venn diagram of three independent IncRNA differential analysis. Blue: RNA sequence data of 3 RCC tissues and paired normal tissues. Red: TCGA-KIRC database. Green: GEO database (GSE64052). b The expression levels of MIR4435-2HG, SNHG12, LINC00645 and LINC01187 in 72 normal tissues and 524 ccRCC tissues in ccRCC based on data from the TCGA database. c The Kaplan-Meier curves of MIR4435-2HG, SNHG12, LINC00645 and LINC01187 in ccRCC for overall survival (OS). d The high expression of SNHG12 was related with various clinicopathological factors: G grade, T stage, lymph node metastasis and distant metastases. e The expression level of SNHG12 was higher in ccRCC tissues than in normal tissues in TCGA database and in Yusenko renal database. The ROC (receiver operating characteristic) curves of SNHG12 (AUC $=0.9651 ; p<0.0001$ ). $\mathbf{f}$ The expression levels of SNHG12 in five RCC cell lines (786-O, A498, ACHN, CAKI-1 and OSRC-2) and normal cell line (293T). The expression levels of SNHG12 in 20 RCC tissues and paired normal tissues. $\mathbf{g}$ Localisation of SNHG12 was assessed by PCR in ACHN and 786-O cells. U6 and GAPDH were used as positive controls for nuclear RNA and cytoplasmic RNA, respectively. $\mathbf{h}$ The distribution of SNHG12 was analysed by FISH in 786-O cells. $18 \mathrm{~S}$ and U6 showed cytoplasm and nucleus, respectively. Scale bars, $10 \mu \mathrm{m} .{ }^{*} P<0.05,{ }^{* *} P<0.01,{ }^{* * *} P<0.001,{ }^{* * *} P<0.0001$. Error bars indicate mean $\pm \mathrm{SD}$.

Table 1 Correlation between SNHG12 mRNA expression and clinicopathological parameters of CCRCC patients.

\begin{tabular}{|c|c|c|c|c|c|}
\hline \multirow[t]{2}{*}{ Parameter } & & \multirow[t]{2}{*}{ Number } & \multicolumn{2}{|c|}{$\begin{array}{l}\text { SNHG12 mRNA } \\
\text { expression }\end{array}$} & \multirow[t]{2}{*}{$P$ value } \\
\hline & & & $\begin{array}{l}\text { Low } \\
(n=250)\end{array}$ & $\begin{array}{l}\text { High } \\
(n=250)\end{array}$ & \\
\hline \multirow[t]{2}{*}{ Age (years) } & $<60$ & 233 & 111 & 122 & 0.370 \\
\hline & $\geq 60$ & 267 & 139 & 128 & \\
\hline \multirow[t]{2}{*}{ Gender } & Female & 173 & 108 & 65 & $<0.001$ \\
\hline & Male & 327 & 142 & 185 & \\
\hline \multirow[t]{2}{*}{ T stage } & $\mathrm{T} 1$ or $\mathrm{T} 2$ & 339 & 184 & 155 & 0.007 \\
\hline & $\mathrm{T} 3$ or $\mathrm{T} 4$ & 161 & 66 & 95 & \\
\hline \multirow[t]{2}{*}{ N stage } & NO or NX & 488 & 248 & 240 & 0.036 \\
\hline & $\mathrm{N} 1$ & 12 & 2 & 10 & \\
\hline \multirow[t]{2}{*}{ M stage } & MO or MX & 447 & 239 & 208 & $<0.001$ \\
\hline & M1 & 53 & 11 & 42 & \\
\hline \multirow[t]{2}{*}{ G grade } & G1 or G2orGx & 246 & 137 & 109 & 0.016 \\
\hline & G3 or G4 & 254 & 113 & 141 & \\
\hline \multirow[t]{2}{*}{ TNM stage } & $I+\|$ & 321 & 176 & 145 & 0.005 \\
\hline & $I I I+I V$ & 179 & 74 & 105 & \\
\hline
\end{tabular}

TNM tumour-node-metastasis, SNHG12 small nucleolar RNA host gene 12, cCRCC clear cell renal cell carcinoma.

gastric cancer ${ }^{23}$, colorectal cancer ${ }^{24}$, prostate cancer ${ }^{25}$ and so on. However, no study has focused on the role of CDCA3 in RCC yet. Thus, we analysed CDCA3 expression differences between RCC tissues and normal tissues in the TCGA-KIRC database. We found that CDCA3 expression was significantly higher in RCC tissues than in normal tissues, and a lower CDCA3 expression level indicated a better prognosis. Correlation analysis between CDCA3 and SNHG12 was performed, and they were found to be significantly positively correlated. Moreover, CDCA3 expression was highly related to numerous clinicopathological characteristics in ccRCC (Fig. 3d, e).
Next, immunohistochemistry (IHC), qRT-PCR and western blotting were performed to further verify that CDCA3 was highly expressed in RCC tissues at both the mRNA and protein levels (Fig. 4f, g). Furthermore, after knocking down or overexpressing CDCA3 (Supplementary Fig. 4a), we studied the biological functions of CDCA3 in RCC cells. According to the results of CCK8 assays, transwell assays and cell cycle analysis, we found that CDCA3 had the capacity to enhance the proliferation, invasion and migration of RCC cells (Supplementary Fig. 4b-f).

\section{SNHG12 promoted tumour progression via CDCA3 in RCC cells}

Here, rescue assays were performed to confirm the roles of the SNHG12/CDCA3 axis in RCC. As described above, 786-O and ACHN cells with SNHG12 stably knocked down or overexpressed were constructed by lentivirus infection. Then, CDCA3 was overexpressed by plasmid in sh SNHG12- and sh NC-treated RCC cells. In SNHG12overexpressing cells and NC cells, CDCA3 expression was low due to CDCA3 siRNA. CCK8 assays showed that CDCA3 overexpression could rescue the negative effect of low SNHG12 expression on cell proliferation in RCC cells. Moreover, CDCA3 siRNA reversed the promotion of SNHG12 on RCC cell proliferation (Fig. 4a). Transwell assays were performed to assess cell migration and invasion. As shown in Fig. 4b, c, the migration and invasion of RCC cells inhibited by sh SNHG12 could be recovered through overexpression of CDCA3. Knocking down CDCA3 could also weaken the positive effect of SNHG12 on RCC cell migration and invasion. For cell cycle assays, we obtained similar results (Fig. 4d, e and Supplementary Fig. 5a, b). Overall, we found that SNHG12 promoted tumour progression via CDCA3 in RCC cells.

\section{SNHG12 increased sunitinib resistance in RCC cells through CDCA3}

The differential expression analysis of lncRNA from GSE64052 already showed that SNHG12 was highly expressed in sunitinib-resistant RCC cells (Supplementary Fig. 6a). The TCGA analysis also revealed that both 
Table 2 Univariate and multivariate analyses of SNHG12 mRNA level and patient survival.

\begin{tabular}{|c|c|c|c|c|c|c|}
\hline \multirow[t]{2}{*}{ Variables } & \multicolumn{3}{|c|}{ Univariate analysis } & \multicolumn{3}{|c|}{ Multivariate analysis $^{c}$} \\
\hline & $H R^{a}$ & $95 \% \mathrm{Cl}^{\mathrm{b}}$ & $P$ value & HR & $95 \% \mathrm{Cl}$ & $P$ value \\
\hline \multicolumn{7}{|l|}{ Overall survival $(n=500)$} \\
\hline SNHG12 low $(n=250)$ high $(n=250)$ & 2.021 & $1.449-2.819$ & 0.000 & 1.510 & $1.060-2.152$ & 0.022 \\
\hline Age (years) $<60(n=233) \geq 60(n=267)$ & 2.011 & $1.429-2.829$ & 0.000 & 1.807 & $1.277-2.555$ & 0.001 \\
\hline Gender female $(n=173)$ male $(n=327)$ & 0.962 & $0.689-1.343$ & 0.821 & - & - & - \\
\hline T stage T1 or T2 $(n=339)$ T3 or T4 $(n=161)$ & 2.963 & $2.147-4.088$ & 0.000 & 1.660 & $1.156-2.384$ & 0.006 \\
\hline $\mathrm{N}$ stage N0 or NX $(n=488) \mathrm{N} 1(n=12)$ & 3.184 & $1.558-6.504$ & 0.001 & 1.520 & $0.724-3.192$ & 0.268 \\
\hline$M$ stage $M 0$ or $M X(n=447) M 1(n=53)$ & 4.855 & $3.410-6.912$ & 0.000 & 2.961 & $1.990-4.404$ & 0.000 \\
\hline $\mathrm{G}$ grade $\mathrm{G} 1$ or $\mathrm{G} 2$ or $\mathrm{GX}(n=246) \mathrm{G} 3$ or $\mathrm{G} 4(n=254)$ & 2.562 & $1.797-3.651$ & 0.000 & 1.806 & $1.246-2.620$ & 0.002 \\
\hline
\end{tabular}

${ }^{a}$ Hazard ratio, estimated from Cox proportional hazard regression model.

${ }^{\mathrm{b}}$ Confidence interval of the estimated HR.

${ }^{c}$ Multivariate models were adjusted for T, N, M classification, age and gender.

SNHG12 and CDCA3 were associated with multidrug resistance (Fig. 5a). Moreover, the PI3K/AKT pathway, a sunitinib-resistant related pathway ${ }^{14,26}$, was correlated with high expression of SNHG12 and CDCA3 (Supplementary Fig. 6b). Therefore, we hypothesised that the SNHG12/CDCA3 axis could play a role in sunitinib resistance. In a previous study, we established two sunitinib-resistant RCC cell lines, 786-O-R and ACHN$\mathrm{R}^{27}$. First, $\mathrm{qRT}-\mathrm{PCR}$ and western blotting assays were conducted to verify that SNHG12 and CDCA3 expression levels were higher in sunitinib-resistant cells (Fig. 5b). Then, the sunitinib sensitivity of ACHN-R was evaluated after SNHG12 knockdown or overexpression. It is clear that SNHG12 expression levels were negatively correlated with drug sensitivity in ACHN-R cells (Fig. 5c). Similarly, we also found that high expression of CDCA3 promoted ACHN-R cell sunitinib resistance (Fig. 5d). As shown in CCK8 assays, sunitinib-resistant RCC cell viability was not affected by $2.5 \mu \mathrm{M}$ concentration of sunitinib when compared with DMSO. However, when SNHG12 or CDCA3 was knocked down, cell viability decreased in the presence of sunitinib (Fig. 5e and Supplementary Fig. 6c). These results revealed that knocking down SNHG12 or CDCA3 might increase the sensitivity of drug-resistant RCC to sunitinib. Nevertheless, under conditions of low SNHG12 expression, overexpressing CDCA3 with plasmid restored sunitinib resistance (Fig. $5 \mathrm{f}$ and Supplementary Fig. 6d). Similarly, in SNHG12-overexpressing cells, when CDCA3 was knocked down with siRNA, the enhanced drug tolerance could be reversed (Fig. $5 \mathrm{~g}$ and Supplementary Fig. 6e). From these findings, we concluded that SNHG12 increased sunitinib resistance in RCC cells through CDCA3.

\section{SNHG12 bound to and stabilised SP1, which activated CDCA3 transcription}

According to published articles, the transcription factors SP1 and HOXB3 could bind to the promoter sequence of $\mathrm{CDCA} 3^{23,25}$. Studies have reported that nucleus-located IncRNAs could regulate protein transcription by binding to transcription factors ${ }^{28,29}$. To assess the possibility of SNHG12 interacting with these transcription factors, the catRAPID algorithm was used. Interestingly, the interaction strength between SNHG12 and SP1 was relatively higher, and potential binding sequences were predicted (Supplementary Fig. 7a, b). Thus, we mainly focused on SP1.

Next, we confirmed the expression promoting effect of SP1 on CDCA3 in RCC cells at the mRNA and protein levels (Fig. 6a, b and Supplementary Fig. 7c). Encouraged by this observation, we predicted the binding sites of SP1 in the CDCA3 promoter with JASPAR (Fig. 6c), and seven potential positions were identified. To validate the exact sites, a chromatin immunoprecipitation (ChIP) assay was performed. In both 786-O and ACHN cells, a strong enrichment between position E2 and anti-SP1 antibody was observed (Fig. 6d and Supplementary Fig. 7d). Furthermore, we constructed a CDCA3 promoter E2-wildtype (WT) GV238 vector and a CDCA3 promoter E2mutant (MUT) GV238 vector. Luciferase activity analysis showed that the luciferase activity of the vector containing the WT CDCA3 promoter could be promoted by SP1 overexpression in 293T cells (Fig. 6e).

In addition, RNA-binding protein immunoprecipitation (RIP) assays were conducted to validate the interaction between SNHG12 and SP1. As shown in Fig. 6f, SNHG12 was significantly enriched by anti-SP1 antibody in 786-O and ACHN cells. However, when SNHG12 expression 


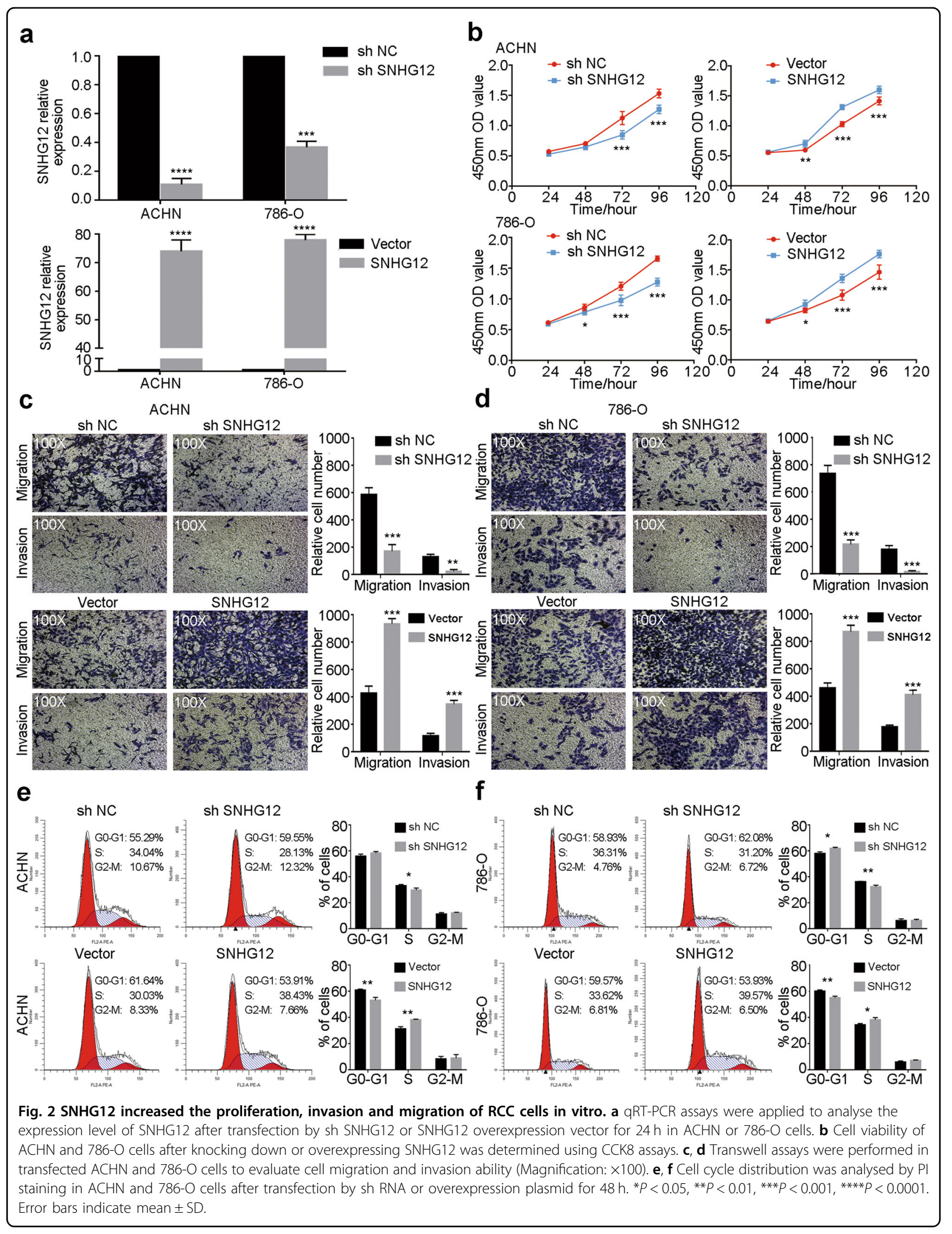




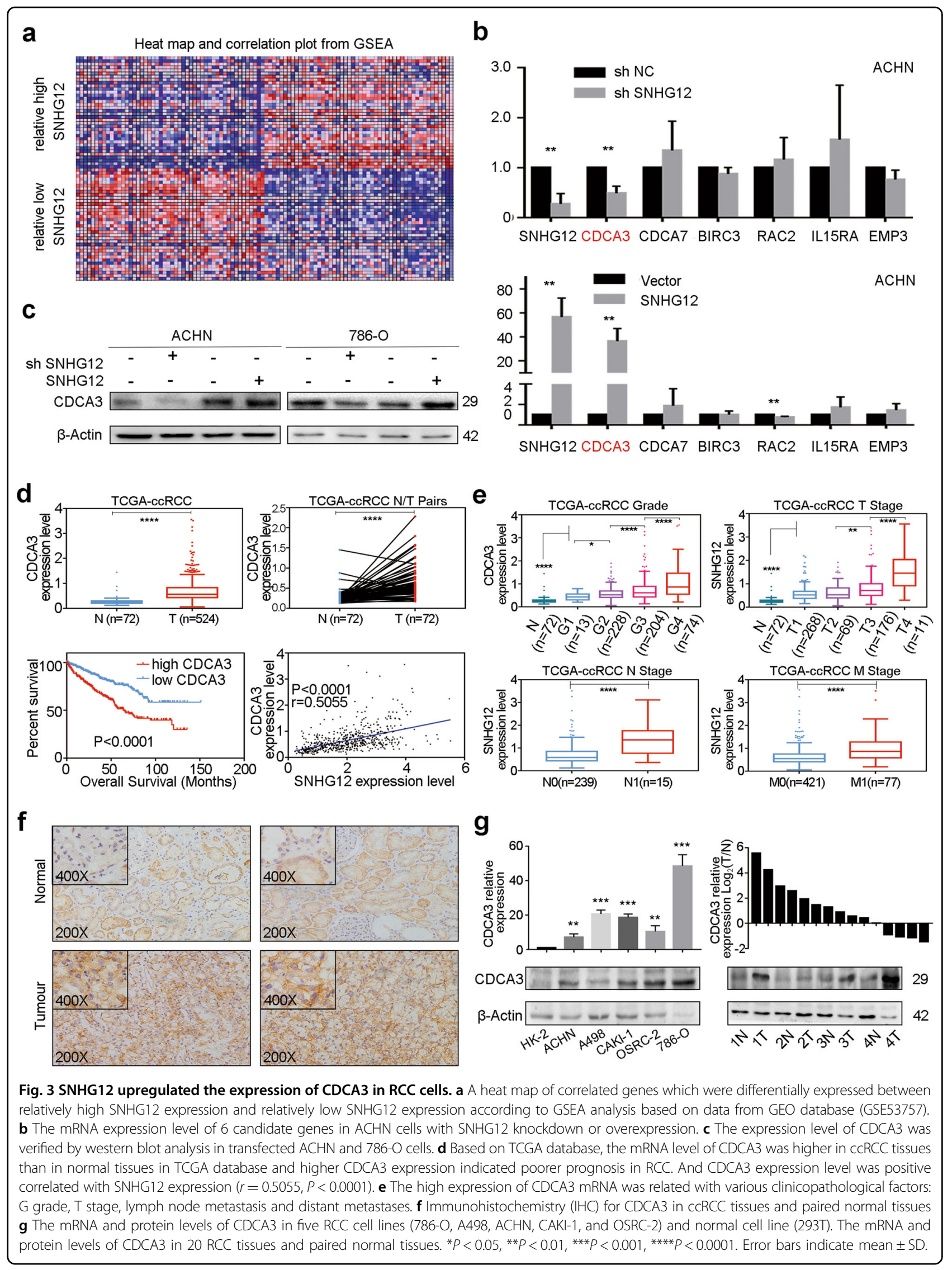




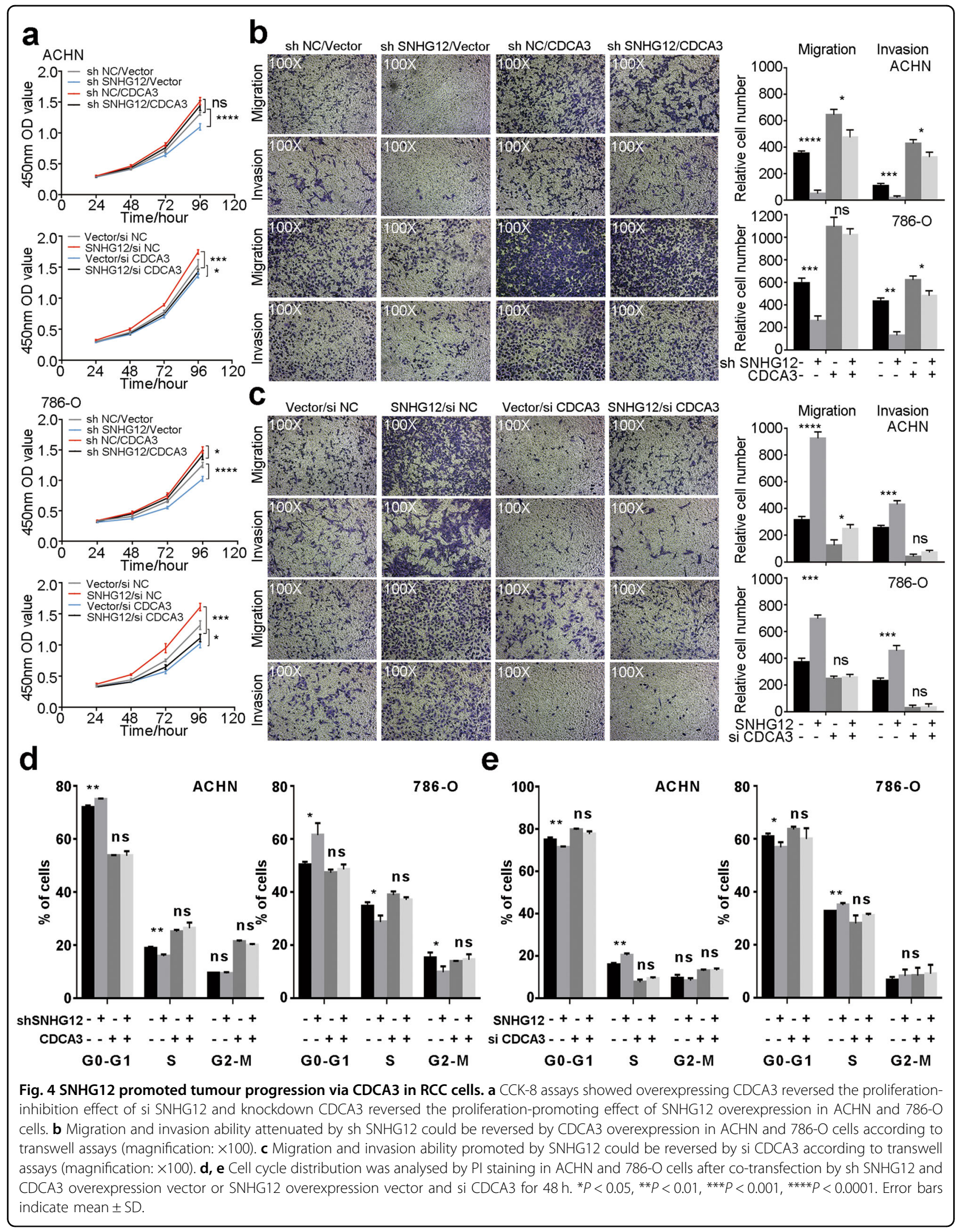




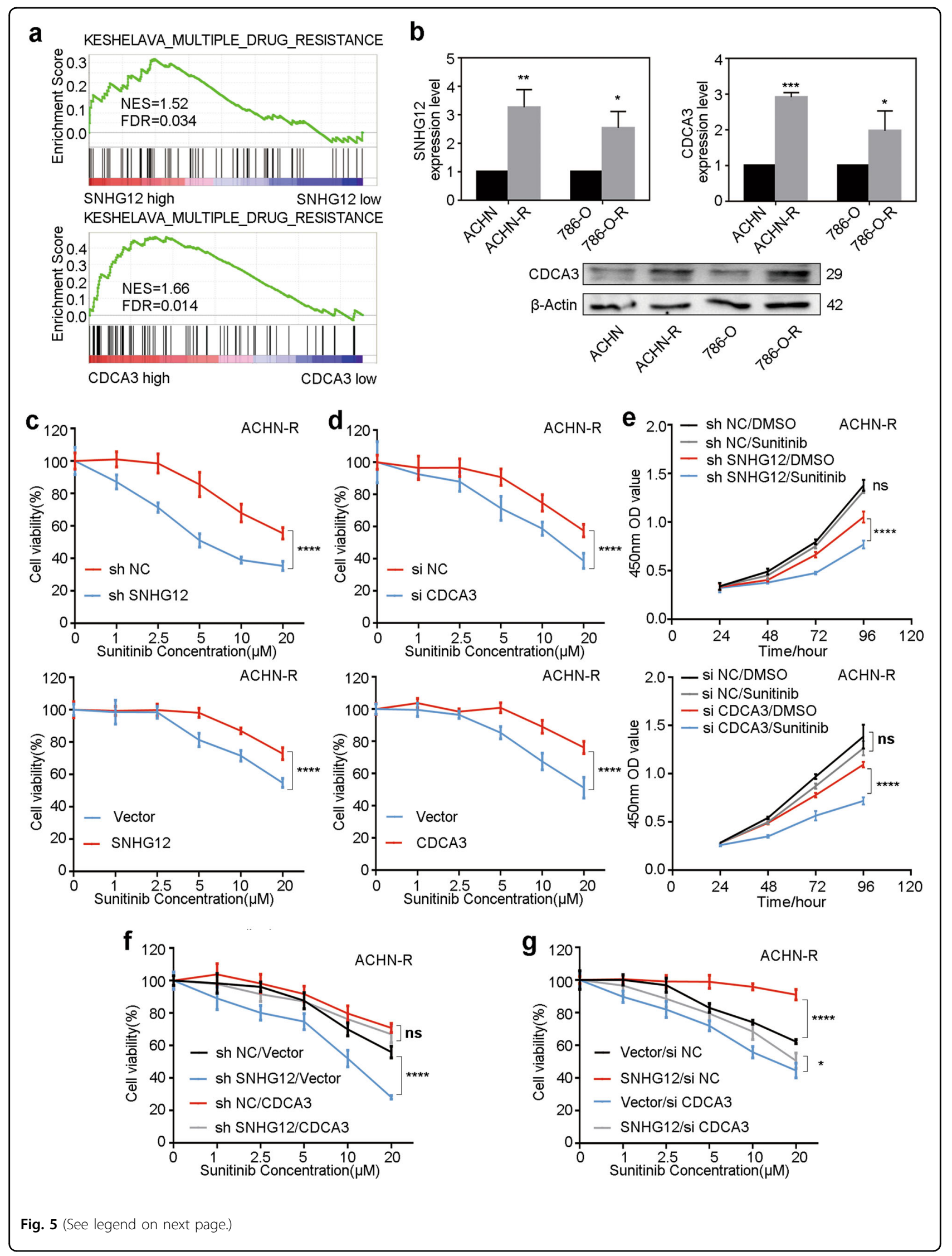


(see figure on previous page)

Fig. 5 SNHG12 increased sunitinib resistance in RCC cells through CDCA3. a GSEA analysis for the correlation of multiple drug resistance and expression level of SNHG12 and CDCA3 according to GEO database (GSE53757. FDR $<0.25, P<0.05$ was considered statistically significant). b qRTPCR was used to determine the expression level of SNHG12 and CDCA3 in sunitinib-resistant RCC cells (ACHN-R and 786-O-R) and in RCC cells (ACHN and 786-O). And CDCA3 protein expression level was detected by western blot assays. c CCK-8 assays were used to measure sunitinib sensitivity of ACHN-R cells after transfection by sh SNHG12 or SNHG12 overexpression vector for $48 \mathrm{~h}$. $\mathbf{d}$ CCK-8 assays were used to measure sunitinib sensitivity of ACHN-R cells after transfection by si CDCA3 or CDCA3 overexpression vector for $48 \mathrm{~h}$. e In ACHN-R cells, knocking down SNHG12 or CDCA3 revered sunitinib resistance, as described by CCK-8 assays. $\mathbf{f}$ The sensitivities of ACHN-R after co-transfection by sh SNHG12 and CDCA3 overexpression vector under different sunitinib concentration were determined by CCK-8 assay. $\mathbf{g}$ The sensitivities of ACHN-R after co-transfection by SNHG12 overexpression vector and si CDCA3 under different sunitinib concentration were determined by CCK-8 assay. ${ }^{*} P<0.05,{ }^{* *} P<0.01,{ }^{* * *} P<0.001$, ${ }^{* * * *} P$ $<0.0001$. Error bars indicate mean $\pm S D$.

was low or high, the SP1 expression level showed inconsistent results at the mRNA and protein levels (Fig. $6 \mathrm{~g}$ ). Some scientists have demonstrated that IncRNAs play a role in post-translational regulation by affecting protein stability $^{28,30}$. Based on this mechanism, cycloheximide (CHX), a protein synthesis inhibitor, was used to investigate whether SNHG12 regulates SP1 stability. Cell protein was isolated from SNHG12 knockdown and negative control RCC cells after CHX treatment for $0 \mathrm{~h}, 8 \mathrm{~h}, 16 \mathrm{~h}$ and $24 \mathrm{~h}$. We found that the protein stability decreased when SNHG12 expression was relatively low (Fig. 6h). Moreover, when SNHG12 was overexpressed, SP1 tended to be more stable (Fig. 6i). As we know, there are different mechanisms of protein degradation, such as the ubiquitin/ proteasomal-mediated pathway and autophagy/lysosomal-mediated pathway ${ }^{31}$. MG132, a proteasome inhibitor, and chloroquine, a lysosome inhibitor, were used to confirm the specific pathway involved in the SP1 stability regulated by SNHG12. As shown in Fig. 6j, when MG132 was added, SP1 remained stable even if SNHG12 was knocked down in RCC cells. Moreover, GO enrichment analysis and GSEA analysis revealed that SNHG12 was involved in numerous ubiquitin-related pathways (Supplementary Fig. 7e). Then, ubiquitination-related immunoprecipitation directly showed that the ubiquitination level of SP1 was regulated by SNHG12 in RCC cells (Fig. 6k). In conclusion, we determined that SNHG12 bound to and stabilised SP1, which activated CDCA3 transcription.

\section{SNHG12 knockdown repressed tumour progression and reversed sunitinib resistance in vivo}

Based on the results above, we studied the function of SNHG12 in vivo. A total of $3 \times 10^{6}$ ACHN-R cells with SNHG12 stable knockdown were implanted subcutaneously into 10 4-week-old nude mice, while the same amount of negative control ACHN-R cells were implanted into 10 other nude mice. From day 20, sunitinib or vehicle was fed to five mice of each group every day. Tumour size was measured every 2 or 3 days starting with tumour formation to day 40. From the results, we observed that knocking down SNHG12 inhibited tumour growth (Fig. 7a). Moreover, in the sh SNHG12 group, both the volume and weight of tumours from mice fed sunitinib were obviously decreased, which revealed that low SNHG12 expression increased RCC sensitivity to sunitinib (Fig. $7 \mathrm{a}-\mathrm{c}$ ). IHC and western blotting were performed in these xenograft tumours. We found that in the sh SNHG12 group, CDCA3 was expressed at low levels (Fig. 7d, e).

In Fig. $7 f$, we generated a schematic diagram of this process. SNHG12 could bind to SP1 and inhibit its ubiquitination and degradation in RCC. In addition, stabilised SP1 promoted the expression of CDCA3, which resulted in RCC progression and sunitinib resistance.

\section{Discussion}

In the present study, we demonstrated that IncRNA SNHG12 plays an essential role in RCC. First, by RNA sequencing of RCC tissues and bioinformatics analysis, we found that SNHG12 expression was higher in RCC tissues and in sunitinib-resistant RCC cells. The higher expression level of SNHG12 was also linked with higher tumour grade and indicated a worse prognosis. Next, functional experiments showed that SNHG12 could promote RCC cell proliferation, migration, invasion and sunitinib resistance. Then, we verified that SNHG12 could regulate the expression of CDCA3 by GSEA analysis and qRT-PCR assays. Furthermore, the SNHG12/CDCA3 axis could promote RCC progression and sunitinib resistance in vitro and in vivo. Mechanically, SNHG12 bound to the transcription factor SP1, which reduced SP1 ubiquitination. The protein stability of SP1 was increased, and as a result, CDCA3 was highly expressed.

Recently, increasing studies have discovered the underlying mechanisms of long noncoding RNAs at multiple regulatory levels ${ }^{32}$. For SNHG12, almost all existing studies agreed that it works as a miRNA sponge in different cancer cells ${ }^{16,18,33,34}$. By this mechanism, SNHG12 functions as an endogenous competitive mimic target of miRNAs, which results in the upregulation of the mRNA that is regulated by these miRNAs. However, in most of these studies, the location of SNHG12 or target miRNA were not discussed, and the target miRNAs were mainly predicted from several specific databases. In our 


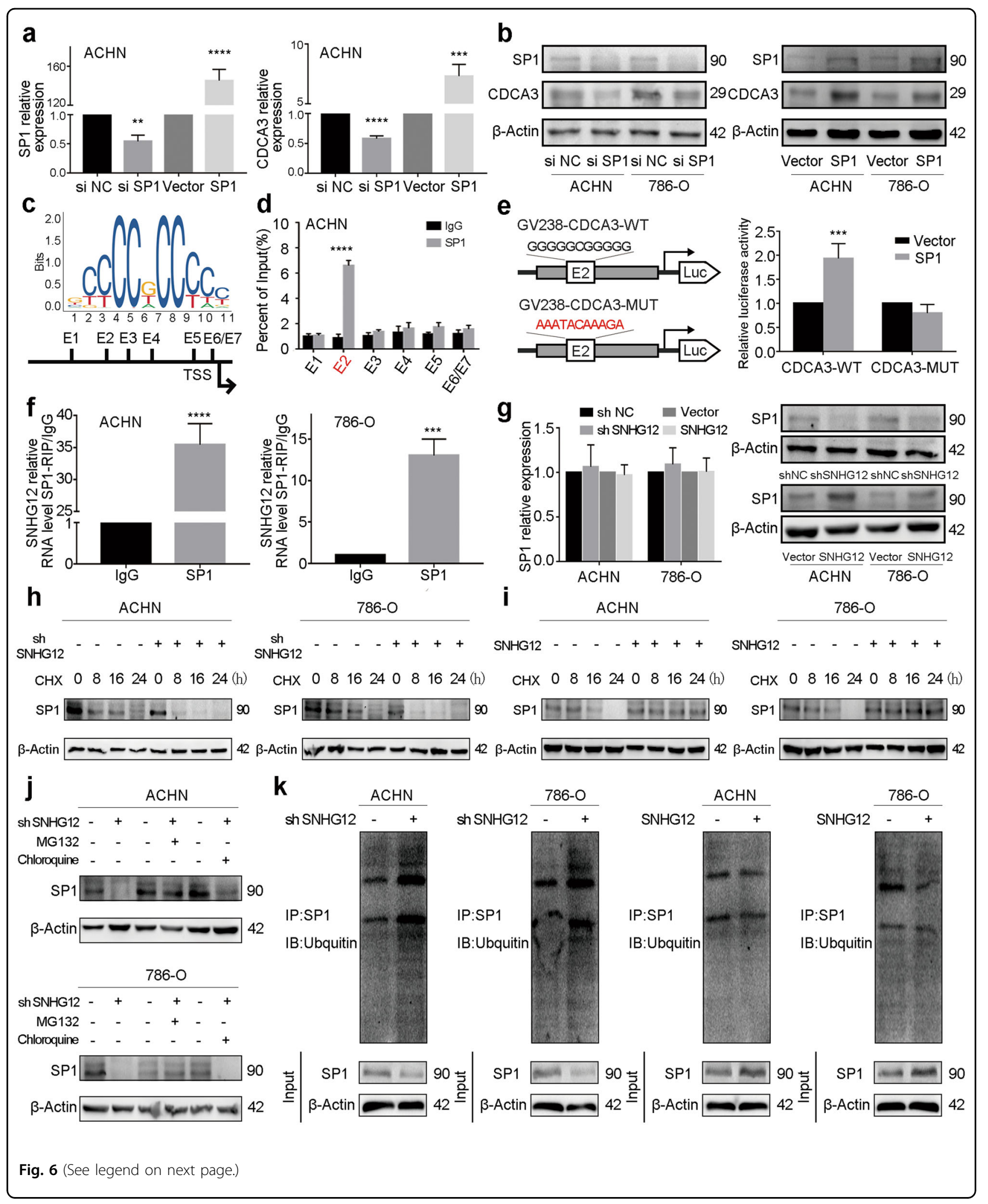


(see figure on previous page)

Fig. 6 SNHG12 bound to and stabilised SP1, which activated CDCA3 transcription. a qRT-PCR for mRNA levels of SP1 and CDCA3 in transfected ACHN cells. $\mathbf{b}$ western blot assays for protein levels of SP1 and CDCA3 in transfected ACHN and 786-O cells. $\mathbf{c}$ The predicted positions of putative SP1 binding motif in -2000-bp human CDCA3 promoter. $\mathbf{d}$ ChIP-PCR assays were performed to show direct binding of SP1 to CDCA3 promoter regions in ACHN cells. e Luciferase reporter assays were performed by co-transfecting the wild type CDCA3 promoter or fragment E2-mutant CDCA3 promoter with SP1 overexpression vector or blank vector in 293T cells. f Anti-SP1 RIP-PCR assays were performed in ACHN and 786-O cells to show SP1 directly bound to SNHG12. g qRT-PCR and western blot for mRNA and protein levels of SP1 in transfected RCC cells. $\mathbf{h}$, i SP1 protein levels were measured by western blot in RCC cells after transfected sh SNHG12 or SNHG12 overexpression vector and treated with cycloheximide (CHX) for a certain period of time. $\mathbf{j}$ Cells with SNHG12 knockdown were treated with vehicle (DMSO), MG132 (20 nM) or chloroquine (50 nM) for $24 \mathrm{~h}$. Western blot assays were applied to show SP1 protein levels. $\mathbf{k}$ Immunoprecipitation with an anti-SP1 antibody were performed in SNHG12 knockdown or overexpression RCC cells, and analysed by western blotting with an anti-ubiquitin antibody. ${ }^{*} P<0.05,{ }^{* *} P<0.01,{ }^{* * *} P<0.001,{ }^{* * *} P<0.0001$. Error bars indicate mean $\pm \mathrm{SD}$.

study, a cellular fractionation assay of RNA and fluorescence in situ hybridisation (FISH) assays were performed, and we confirmed that SNHG12 is mainly located in the nucleus. This result was consistent with the findings from Sun et al. ${ }^{35}$. On the basis of this result, we assumed that SNHG12 could play a regulatory role at the transcriptional level in the nucleus. Previously, Jiang et al. demonstrated that SNHG15, a member of the SNHG family, interacted with and stabilised the transcription factor Slug and promoted colon cancer progression ${ }^{28}$. Post-translational modification is an important and common regulatory mechanism in proteomics, and lncRNAs are involved in various types of comprehensive modifications, such as phosphorylation ${ }^{36}$, ubiquitination ${ }^{37}$ and acetylation ${ }^{38}$. The SP1 transcription factor, an essential transcription factor, is involved in many cellular processes. It could be activated or repressed by different post-translational modifications, including phosphorylation, ubiquitination and glycosylation ${ }^{39}$. Wang et al. clarified that RNF4 acted as the E3 ubiquitin ligase and initiated ubiquitin-mediated proteolysis of SP1 $1^{40}$. In our study, RIP assays demonstrated that SNHG12 directly bound to SP1 in RCC cells. Subsequent protein stability assays showed that highly expressed SNHG12 stabilised the SP1 protein. Furthermore, we could clearly see by coimmunoprecipitation assay that more ubiquitin bound to SP1 when SNHG12 was knocked down. Hence, we found that SNHG12 could inhibit SP1 ubiquitin-mediated proteolysis in RCC cells, which was a completely different regulatory mechanism of SNHG12 from previous studies.

CDCA3 is a protein that has not been researched deeply. Published articles about CDCA3 mainly focused on the role of CDCA3 in cell cycle regulation and drug resistance in digestive tumours and lung cancer ${ }^{24,41}$. It has been reported that CDCA3 functions as an F-box protein that leads to the ubiquitylation-dependent proteolysis of Wee1 and ultimately results in irreversible mitotic entry ${ }^{42}$. In our research, we found for the first time that CDCA3 upregulated by SNHG12/SP1 was related to RCC and was involved in sunitinib resistance. SNHG12/SP1/CDCA3 might increase the cell cycle transition from G1 phase to S phase and promote cell proliferation in combination with sunitinib. However, the specific mechanism of SNHG12/ SP1/CDCA3 regulating sunitinib resistance in RCC remains unclear and needs further studies.

There are limited therapeutic options for advanced metastatic RCC patients. With the use of receptor tyrosine kinase inhibitors (RTKIs), including sunitinib and sorafenib, the overall survival of these patients has been prolonged considerably. Unfortunately, the majority of patients develop sunitinib resistance after 6-15 months, which eventually leads to tumour progression ${ }^{5-7}$. Hence, it is urgent to discover potential mechanisms and identify biomarkers of sunitinib resistance. Studies have revealed that compensatory negative feedback in receptor tyrosine kinase pathways is an important mechanism of adaptive resistance to targeted therapy of cancer ${ }^{43}$. As the downstream effector of VEGFR, the PI3K/AKT signalling pathway was reported to be activated in drug-resistant cells $^{14}$. Furthermore, a phase II study of combination therapy with perifosine, an inhibitor of AKT, and sorafenib in patients with lymphoproliferative diseases has already been completed ${ }^{44}$. In this study, we found that lncRNA SNHG12 was highly expressed in sunitinibresistant RCC cells. SNHG12 attenuated sunitinib sensitivity by promoting CDCA3 expression via the transcription factor SP1. In addition, by GSEA analysis, we discovered that the PI3K/AKT signalling pathway was positively correlated with SNHG12 and CDCA3, which suggested that the SNHG12/SP1/CDCA3 axis might promote sunitinib resistance via the PI3K/AKT signalling pathway. However, more detailed studies are needed to explore the specific mechanism.

In summary, we demonstrated for the first time that long noncoding RNA SNHG12 could upregulate the expression of CDCA3 by stabilising the transcription factor SP1 and thereby promoting tumour progression and sunitinib resistance in RCC. Our findings in this study could provide a novel diagnostic and therapeutic target for RCC. 


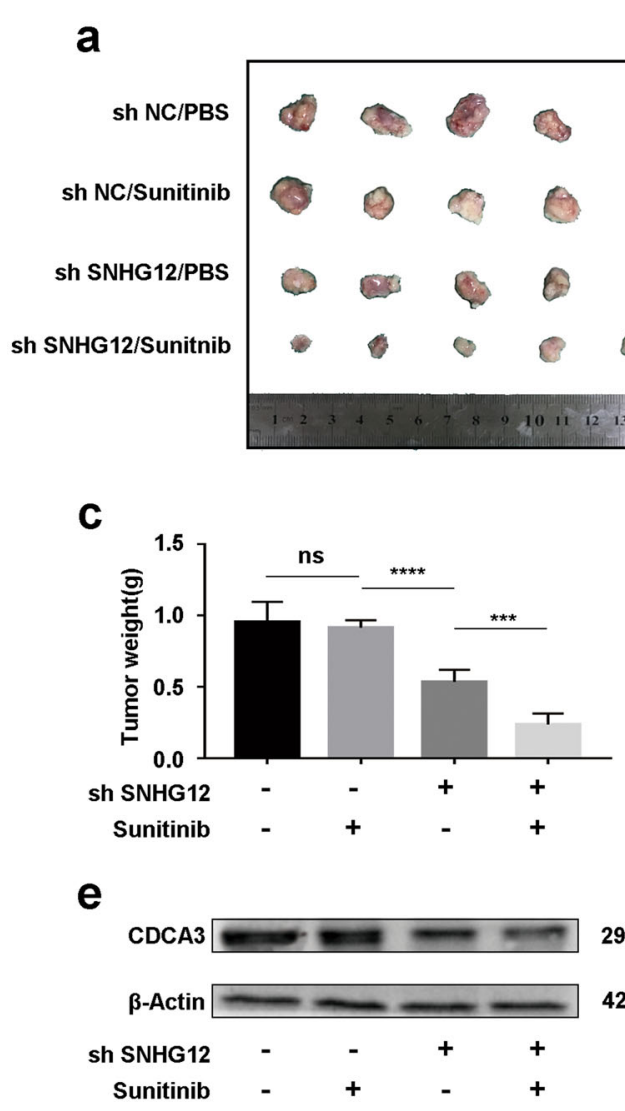

b

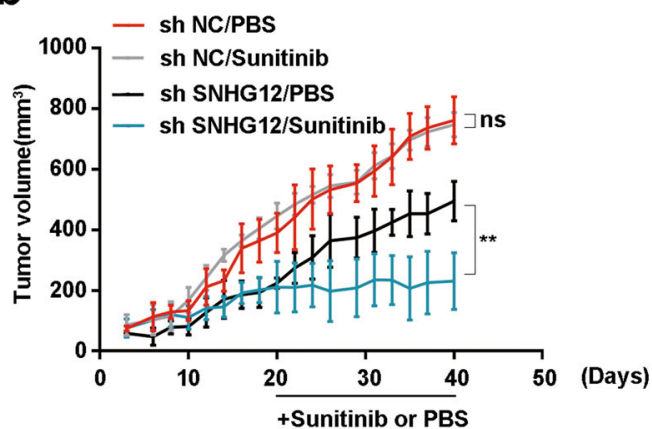

d

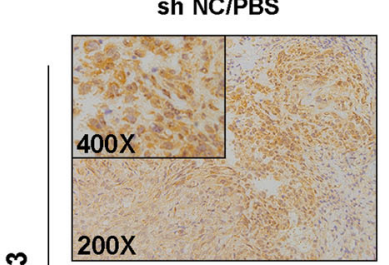

sh NC/Sunitinib

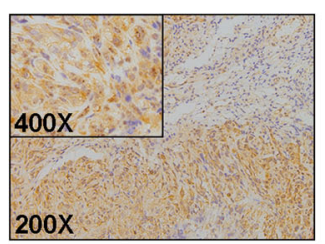

sh SNHG12/PBS

sh SNHG12/Sunitinib
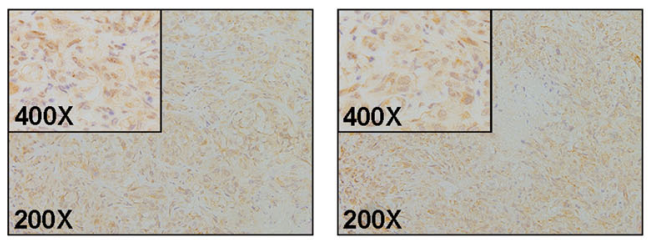

f

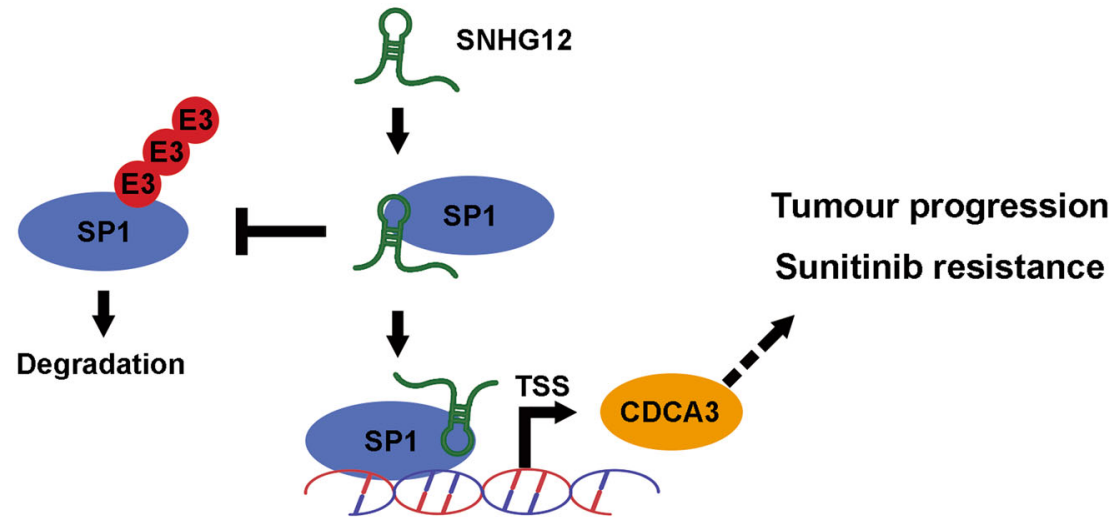

Fig. 7 SNHG12 knockdown repressed tumour progression and reversed sunitinib resistance in vivo. a ACHN-R cells stably knockdown of SNHG12 were subcutaneously injected into nude mice. b, $\mathbf{c}$ The volume and weight of xenograft tumours in four groups with different treatment $(n=5)$. d, e Immunohistochemistry $(\mathrm{IHC})$ and western blot assays were performed to measure CDCA3 expression levels in four groups with different treatment. f Schematic diagram illustrated IncRNA SNHG12 regulated SNHG12/SP1/CDCA3 axis promoting tumour progression and sunitinib resistance in $\mathrm{RCC} .{ }^{*} P<0.05,{ }^{* *} P<0.01,{ }^{* * *} P<0.001,{ }^{* * *} P<0.0001$. Error bars indicate mean \pm SD.

\section{Materials and methods}

Human RCC tissue samples and cell culture

A total of 50 matched RCC and adjacent normal renal tissues were obtained from the Department of Urology, Wuhan Union Hospital between September 2016 and
September 2018. The present study was approved by the Ethics Committee of Human Research of Huazhong University of Science and Technology (HUST), and written informed consent was obtained from patients prior to surgery.Human RCC cell lines (786-O, ACHN, 
A498, OSRC-2 and Caki-1) and the human renal proximal tubular epithelial cell line HK-2 were purchased from the American Type Culture Collection (ATCC) in the USA. These cells were cultured in high-glucose DMEM containing 10\% foetal bovine serum and 1\% streptomycinpenicillin (Google Biotechnology, Wuhan, China) and maintained in a humidified atmosphere with $5 \% \mathrm{CO} 2$ at $37^{\circ} \mathrm{C}$.

\section{Transient transfection and lentivirus infection assay}

Short hairpin RNA (shRNA) against SNHG12 (sh SNHG12) and a negative control shRNA (sh NC) were designed and synthesised by Vigene (Vigene Biology, Shangdong, China). The small interfering RNAs (siRNAs) si CDCA3, si SP1 and si NC (cat. no. siN0000002-1-5) were obtained from Guangzhou RiboBio (RiboBio, Guangzhou, China). The RNA sequences used for transfection in this study are shown in Supplementary Table 2. In addition, the pcDNA3.1 vector (Vigene Biology) containing the full-length cDNA sequence of SNHG12, CDCA3 or SP1 was used to overexpress SNHG12, CDCA3 or SP1, respectively. The empty pcDNA3.1 vector was used as a negative control. For cell transient transfection, Lipofectamine 2000 reagent (Invitrogen, Carlsbad, CA) was used according to the manufacturer's instructions when the RCC cell lines were at $70-80 \%$ confluence.

The lentivirus pLent-shSNHG12-GFP-Puro or its negative control (NC) pLent-GFP-Puro (Vigene Biology) was used to infect RCC cells with enhanced infection solution (Vigene Biology) according to the manufacturer's protocol. Similarly, pLent-SNHG12-GFP-Puro lentivirus or empty vector (vector) pLent-GFP-Puro lentivirus (Vigene Biology) was used to overexpress genes. Seventytwo hours after the cells were infected with lentivirus, $2 \mu \mathrm{g} / \mathrm{ml}$ puromycin was added to kill the cells that had not been transfected.

\section{Quantitative reverse transcription PCR}

Total RNA was isolated from tissues or cells using TRIzol Reagent (Thermo Fisher Scientific, Massachusetts, USA). Then, the extracted RNA was reverse transcribed into cDNA using a Superscript II reverse transcription kit (Takara Bio, Beijing, China) according to the manufacturer's protocols. Subsequently, qRT-PCR was conducted with a SYBR-Green master kit (Vazyme, Nanjing, China) on a LightCycler 480 II (Roche Diagnostics) instrument according to the manufacturer's protocols. The primers used to amplify SNHG12, CDCA3, CDCA7, BIRC3, RAC2, IL15RA, EMP3, SP1 and GAPDH were chemically synthesised by TSINGKE (TSINGKE, Beijing, China). All qRT-PCR reactions were performed in triplicate. The primer sequences used for qRT-PCR in this study are shown in Supplementary Table 3.

\section{Cellular fractionation assay of RNA}

The cellular fraction assay was performed using a PARIS $^{\mathrm{TM}}$ Kit (Invitrogen, USA) according to the manufacturer's instructions. GAPDH mRNA was used as a cytoplasmic control, and U6 RNA was used as a nuclear control. The fractionated RNA was examined by qRT PCR in triplicate.

\section{RNA fluorescence in situ hybridisation}

Cy3-labelled SNHG12 probes as well as Cy3-labelled U6 and $18 \mathrm{~S}$ probes used as positive controls for the nucleus and cytoplasm, respectively, were purchased from RiboBio. Hybridisation was performed with a Fluorescent in Situ Hybridization kit (RiboBio) according to the manufacturer's instructions. Slides were observed using an IX71 inverted microscope (Olympus, Japan). RNA FISH experiments were performed in the 786-O and $\mathrm{ACHN}$ cell lines.

\section{Western blotting}

Proteins from tissues and cells were extracted using RIPA buffer (Servicebio, Wuhan, China) containing protease inhibitors. Then, protein concentrations were determined using a BCA Protein Assay kit (Beyotime Institute of Biotechnology). A total of $30 \mu \mathrm{g}$ of protein was subjected to $10 \%$ SDS-PAGE and transferred to a polyvinylidene difluoride (PVDF) membrane (EMD Millipore, Bedford, USA). PVDF membranes were then blocked in $5 \%$ skim milk for $2 \mathrm{~h}$. Subsequently, samples were incubated with specific primary antibodies against CDCA3, SP1, ubiquitin and $\beta$-actin at $4{ }^{\circ} \mathrm{C}$ overnight. Next, membranes were incubated with the appropriate secondary antibodies for $2 \mathrm{~h}$ at room temperature. Finally, the protein bands were visualised with Pierce ${ }^{\mathrm{TM}}$ ECL Western Blotting Substrate (Thermo Fisher Scientific) using ChemiDoc XRS+(Bio-Rad Laboratories, Inc.). Information on the antibodies is shown in Supplementary Table 4.

\section{Co-immunoprecipitation}

Forty-eight hours after transfection, the cells were lysed in Triton lysis buffer. After that, $20 \mu \mathrm{L}$ of protein A/G PLUS-Agarose (Santa Cruz, CA, USA) together with antiSP1 antibody or control IgG antibody were added to the supernatant of centrifuged lysates and incubated overnight at $4{ }^{\circ} \mathrm{C}$. The next day, protein A/G PLUS-Agarose was precipitated and washed with Triton lysis buffer three times. Finally, 2X SDS sample buffer was added to the immunoprecipitates, and the samples were subjected to western blotting after boiling for $10 \mathrm{~min}$.

\section{Chromatin immunoprecipitation}

ChIP assays were conducted following the protocol of the EZ-ChIP ${ }^{\mathrm{TM}}$ kit (Millipore, Billerica, MA). Chromatin 
was sonicated to obtain 150-900-bp DNA/protein fragments after cells were cross-linked with formaldehyde. Then, anti-SP1 or control antibody was added to lysates and incubated at $4{ }^{\circ} \mathrm{C}$ overnight. The next day, ChIPGrade Protein G Agarose Beads were used for immunoprecipitation. After that, DNA was released and purified for subsequent qRT-PCR.

\section{RNA-binding protein immunoprecipitation (RIP)}

RIP assays were performed following the protocol of the RIP $^{\mathrm{TM}}$ RNA binding protein immunoprecipitation kit (Millipore). Cells were lysed with complete RIP lysis buffer. Then, anti-SP1 antibody or control IgG antibody was incubated with magnetic beads for $30 \mathrm{~min}$. After that, the beads were added to lysates and incubated at $4{ }^{\circ} \mathrm{C}$ overnight. Finally, RNA was purified for subsequent qRTPCR assay.

\section{Luciferase reporter assays}

The sequence of the CDCA3 promoter sites $(-2000$ to transcription initiation site) containing the wild-type binding sequence or mutant type binding sequence was cloned in a GV238-based vector. 293T cells were transfected with the GV238-CDCA3-WT or GV238-CDCA3MUT plasmid. After $24 \mathrm{~h}$, cells were lysed, and luciferase activity was measured with a Dual-Luciferase Reporter Assay Kit (Promega, Madison, WI, USA). Renilla luciferase was used for normalisation. All transfections were repeated in triplicate.

\section{Immunohistochemistry assay}

Briefly, tissues obtained from ccRCC patients or tumour xenograft mice were sequentially fixed in formalin at room temperature for $12 \mathrm{~h}$, dehydrated and embedded in paraffin. Then, IHC was conducted with rabbit antibodies against CDCA3 (1:100, Abcam, Cambridge, UK). Finally, tissue slides were observed in three randomly selected fields under a light microscope (Olympus Corporation) at $\times 5$ and $\times 200$ magnification.

\section{Cell proliferation assay}

The proliferation ability of ACHN and 786-O cells was assessed with the Cell Counting Kit-8 (Dojindo Molecular Technologies, Kyushu, Japan) reagent according to the manufacturer's instructions. Cells were inoculated on 96well plates at a density of $1 \times 10^{3}$ cells per well with $100 \mu \mathrm{l}$ of medium. Every $24 \mathrm{~h}$ for a total of $96 \mathrm{~h}$, CCK8 solution $(10 \mu \mathrm{l})$ was added to each well, and the cells were further incubated at $37^{\circ} \mathrm{C}$ for $3 \mathrm{~h}$. The absorbance of each well was measured at $450 \mathrm{~nm}$ with a spectrophotometer.

\section{Flow cytometry cell cycle assay}

After transient transfection, $\mathrm{ACHN}$ and $786-\mathrm{O}$ cells were fixed in $75 \%$ ethanol for $12 \mathrm{~h}$. Then, cells were stained with propidium iodide (Beyotime) for cell cycle analysis. Finally, the percentage of cells in each cell cycle phase (G0/G1, S and G2/M) was assessed, and the results were analysed by ModFit LT software.

\section{Cell migration and invasion assays}

Migration and invasion assays were performed as previously described ${ }^{45}$. After starving the cells for $6-8 \mathrm{~h}$ in serum-free DMEM, a total of $1 \times 10^{4}$ cells were seeded in the upper chamber with $200 \mu \mathrm{l}$ of serum-free medium for the migration assay. In addition, $2 \times 10^{4}$ cells were added into Matrigel-coated upper Transwell chambers for the invasion assay. The lower chambers were filled with DMEM containing $10 \% \mathrm{FBS}$. After incubation at $37^{\circ} \mathrm{C}$ for $24 \mathrm{~h}$, cells on the lower surface of the membrane were fixed in $100 \%$ methanol and stained with $0.1 \%$ crystal violet dye for $20 \mathrm{~min}$ at room temperature. Finally, after washing with PBS, cells were imaged in five randomly selected fields under a light microscope (Olympus Corporation) at $\times 100$ magnification.

\section{Tumour xenograft model}

Four-week-old male BALB/c nude mice (HFK Biotechnology, Beijing, China) were chosen for the tumour xenograft model. A total of $3 \times 10^{6}$ ACHN-R cells stably transfected with sh SNHG12 or sh NC were subcutaneously injected into the upper back of each mouse ( $n=10 \mathrm{mice} /$ group). Tumour sizes were measured every 2 or 3 days. After 20 days, 5 mice in the sh SNHG12 group and 5 mice in the sh NC group were fed sunitinib, while the remaining mice in the two groups were fed vehicle. Mice were killed after 40 days. Tumour size was measured, and IHC assays were conducted. The animal experiments were approved by the Animal Ethics Committee of HUST.

\section{Bioinformatics analysis}

SNHG12 expression levels in ccRCC specimens and correlated clinical data were downloaded from The Cancer Genome Atlas database (TCGA; https://xenabrowser. net/heatmap/), GEO database (GSE64052, GSE53757; https://www.ncbi.nlm.nih.gov/geo/) and Oncomine database (Yusenko Renal dataset; https://www.oncomine.org). The gene set enrichment analysis (GSEA; http://software. broadinstitute.org/gsea/index.jsp) was performed to determine signalling pathways and molecules involved in the pathogenesis of ccRCC when SNHG12 was highly expressed.

\section{Statistical analysis}

In the present study, statistical analyses were performed by GraphPad Prism 7.0 (GraphPad Software, San Diego, California, USA) and SPSS 22.0 (IBM, NY, USA). Student's $t$ test or paired Student's $t$ test, receiver operator 
characteristic curve, Pearson $\chi^{2}$ test, Cox regression analysis, linear regression and Kaplan-Meier curve with logrank test were conducted as indicated. Significance was determined at $P<0.05$.

\section{Acknowledgements}

This study was supported by the National Key R\&D Program of China (grant nos. 2017YFB1303100), the National Natural Science Foundation of China (grant nos. 81672524, 81672528 and 81874090), the Hubei Provincial Natural Science Foundation of China (grant no. 2018CFA038), the Independent Innovation Foundation of Huazhong University of Science and Technology (grant no. 118530309), the Clinical Research Physician Program of Tongji Medical College, Huazhong University of Science and Technology (grant no. 5001530015) and the Integrated Innovation Team for Major Human Disease Program of Tongji Medical College, Huazhong University of Science and Technology.

\section{Author details}

'Department of Urology, Union Hospital, Tongji Medical College, Huazhong University of Science and Technology, No. 1277 Jiefang Avenue, 430022 Wuhan, China. ${ }^{2}$ Department of Breast and Thyroid Surgery, Union Hospital, Tongji Medical College, Huazhong University of Science and Technology, No. 1277 Jiefang Avenue, 430022 Wuhan, China. ${ }^{3}$ Department of Pathogenic Biology, School of Basic Medicine, Huazhong University of Science and Technology, No. 13 Hangkong Road, 430030 Wuhan, China

\section{Conflict of interest}

The authors declare that they have no conflict of interest.

\section{Publisher's note}

Springer Nature remains neutral with regard to jurisdictional claims in published maps and institutional affiliations.

Supplementary Information accompanies this paper at (https://doi.org/ 10.1038/s41419-020-2713-8).

Received: 3 April 2020 Revised: 9 June 2020 Accepted: 15 June 2020 Published online: 08 July 2020

\section{References}

1. Moch, H., Cubilla, A. L., Humphrey, P. A., Reuter, V. E. \& Ulbright, T. M. The 2016 WHO Classification of tumours of the urinary system and male genital organspart A: renal, penile, and testicular tumours. Eur. Urol. 70, 93-105 (2016).

2. Rini, B. I., Campbell, S. C. \& Escudier, B. Renal cell carcinoma. Lancet 373, 1119-1132 (2009).

3. Siegel, R. L., Miller, K. D. \& Jemal, A. Cancer statistics, 2019. CA Cancer J. Clin. 69 7-34 (2019).

4. Cohen, H. T. \& McGovern, F. J. Renal-cell carcinoma. N. Engl. J. Med. 353 , 2477-2490 (2005).

5. Bergers, G. \& Hanahan, D. Modes of resistance to anti-angiogenic therapy. Nat. Rev. Cancer 8, 592-603 (2008).

6. Molina, A. M. et al. Sunitinib objective response in metastatic renal cell carcinoma: analysis of 1059 patients treated on clinical trials. Eur. J. Cancer 50, 351-358 (2014).

7. Motzer, R. J. et al. Sunitinib versus interferon alfa in metastatic renal-cell carcinoma. New Engl. J. Med. 356, 115-124 (2007).

8. Mattick, J. S. Non-coding RNAs: the architects of eukaryotic complexity. EMBO Rep. 2, 986-991 (2001).

9. Rinn, J. L. et al. Functional demarcation of active and silent chromatin domains in human HOX loci by noncoding RNAs. Cell 129, 1311-1323 (2007).

10. Tsai, M. C. et al. Long noncoding RNA as modular scaffold of histone modification complexes. Science 329, 689-693 (2010).

11. Yang, $L$. et al. IncRNA-dependent mechanisms of androgen-receptorregulated gene activation programs. Nature 500, 598-602 (2013).

12. Hung, C. L. et al. A long noncoding RNA connects C-Myc to tumor metabolism. Proc. Natl Acad. Sci. USA 111, 18697-18702 (2014).
13. Prensner, J. R. et al. The long noncoding RNA SChLAP1 promotes aggressive prostate cancer and antagonizes the SWISNF complex. Nat. Genet. 45, 1392-1398 (2013).

14. $\mathrm{Qu}$, L. et al. Exosome-transmitted IncARSR promotes sunitinib resistance in renal cancer by acting as a competing endogenous RNA. Cancer Cell 29, 653-668 (2016).

15. Tamang, S. et al. SNHG12: an LncRNA as a potential therapeutic target and biomarker for human cancer. Front. Oncol. 9, 901 (2019).

16. Cheng, G. et al. Long noncoding RNA SNHG12 indicates the prognosis of prostate cancer and accelerates tumorigenesis via sponging miR-133b. J. Cell. Physiol. 235, 1235-1246 (2020).

17. Wang, $Y$., Liang, S., Yu, Y., Shi, Y. \& Zheng, H. Knockdown of SNHG12 suppresses tumor metastasis and epithelial-mesenchymal transition via the Slug/ZEB2 signaling pathway by targeting miR-218 in NSCLC. Oncol. Lett. 17, 2356-2364 (2019).

18. Zhou, B., Li, L., Li, Y., Sun, H. \& Zeng, C. Long noncoding RNA SNHG12 mediates doxorubicin resistance of osteosarcoma via miR-320a/MCL1 axis. Biomed. Pharmacother. 106, 850-857 (2018).

19. Zhu, L. et al. c-Myc mediated upregulation of long noncoding RNA SNHG12 regulates proliferation and drug sensitivity in natural killer/T-cell lymphoma. J. Cell. Biochem. 120, 12628-12637 (2019).

20. Zhang, L. et al. Anti-S1P antibody as a novel therapeutic strategy for VEGFR TKI-resistant renal cancer. Clin. Cancer Res. 21, 1925-1934 (2015).

21. Yusenko, M. V. et al. High-resolution DNA copy number and gene expression analyses distinguish chromophobe renal cell carcinomas and renal oncocytomas. BMC Cancer 9, 152 (2009).

22. von Roemeling, C. A. et al. Neuronal pentraxin 2 supports clear cell renal cell carcinoma by activating the AMPA-selective glutamate receptor-4. Cancer Res. 74, 4796-4810 (2014)

23. $Y u, J$. et al. DNA hypomethylation promotes invasion and metastasis of gastric cancer cells by regulating the binding of SP1 to the CDCA3 promoter. J. Cell. Biochem. 121, 142-151 (2020).

24. Qian, W. et al. CDCA3 mediates p21-dependent proliferation by regulating E2F1 expression in colorectal cancer. Int J. Oncol. 53, 2021-2033 (2018).

25. Chen, J. et al. HoxB3 promotes prostate cancer cell progression by transactivating CDCA3. Cancer Lett. 330, 217-224 (2013).

26. Huang, J., Wang, X., Wen, G. \& Ren, Y. miRNA2055p functions as a tumor suppressor by negatively regulating VEGFA and PIJK/Akt/mTOR signaling in renal carcinoma cells. Oncol. Rep. 42, 1677-1688 (2019).

27. Ruan, H. et al. Enhanced expression of caveolin-1 possesses diagnostic and prognostic value and promotes cell migration, invasion and sunitinib resistance in the clear cell renal cell carcinoma. Exp. Cell Res. 358, 269-278 (2017).

28. Jiang, $\mathrm{H}$. et al. Long non-coding RNA SNHG15 interacts with and stabilizes transcription factor Slug and promotes colon cancer progression. Cancer Lett. 425, 78-87 (2018).

29. Long, Y. C., Wang, X. Y., Youmans, D. T. \& Cech, T. R. How do IncRNAs regulate transcription? Sci. Adv. 3, eaao2110 (2017).

30. $\mathrm{Yu}$, T. et al. MetaLnc9 facilitates lung cancer metastasis via a PGK1-activated AKT/mTOR pathway. Cancer Res. 77, 5782-5794 (2017).

31. Korolchuk, V. I., Menzies, F. M. \& Rubinsztein, D. C. Mechanisms of cross-talk between the ubiquitin-proteasome and autophagy-lysosome systems. FEBS Lett. 584, 1393-1398 (2010).

32. Zhang, X. et al. Mechanisms and functions of long non-coding RNAs at multiple regulatory levels. Int. J. Mol. Sci. https://doi.org/10.3390/ijms20225573 (2019).

33. Lan, T. et al. Long non-coding RNA small nucleolar RNA host gene 12 (SNHG12) promotes tumorigenesis and metastasis by targeting miR-199a/b5p in hepatocellular carcinoma. J. Exp. Clin. Cancer Res. 36, 11 (2017).

34. Yin, W. L., Yin, W. G., Huang, B. S. \& Wu, L. X. LncRNA SNHG12 inhibits miR-199a to upregulate SIRT1 to attenuate cerebral ischemia/reperfusion injury through activating AMPK signaling pathway. Neurosci. Lett. 690, 188-195 (2019).

35. Sun, Y. et al. Long noncoding RNA SNHG12 facilitates the tumorigenesis of glioma through miR-101-3p/FOXP1 axis. Gene 676, 315-321 (2018).

36. Wang, P. et al. The STAT3-binding long noncoding RNA Inc-DC controls human dendritic cell differentiation. Science 344, 310-313 (2014).

37. Ni, W. et al. A novel IncRNA uc.134 represses hepatocellular carcinoma progression by inhibiting CUL4A-mediated ubiquitination of LATS1. J. Hematol. Oncol. 10, 91 (2017).

38. Chen, R. et al. Quantitative proteomics reveals that long non-coding RNA MALAT1 interacts with DBC1 to regulate p53 acetylation. Nucleic Acids Res. $\mathbf{4 5}$ 9947-9959 (2017). 
39. Chang, W. C. \& Hung, J. J. Functional role of post-translational modifications of Sp1 in tumorigenesis. J. Biomed. Sci. 19, 94 (2012).

40. Wang, Y. T., Yang, W. B., Chang, W. C. \& Hung, J. J. Interplay of posttranslational modifications in Sp1 mediates Sp1 stability during cell cycle progression. $J$ Mol. Biol. 414, 1-14 (2011).

41. O'Byrnel, K., Adams, M., Burgess, J. \& Richard, D. CDCA3 regulates the cell cycle and modulates cisplatin sensitivity in non-small cell lung cancer. J. Thorac. Oncol. 11, S65-S65 (2016).

42. Qiu, C. et al. F-box proteins Pof3 and Pof1 regulate Wee1 degradation and mitotic entry in fission yeast. J. Cell Sci. https://doi.org/10.1242/jcs.202895 (2018).
43. Heng, D. Y. et al. Primary anti-vascular endothelial growth factor (VEGF)refractory metastatic renal cell carcinoma: clinical characteristics, risk factors, and subsequent therapy. Ann. Oncol. 23, 1549-1555 (2012).

44. Guidetti, A. et al. Phase II study of perifosine and sorafenib dual-targeted therapy in patients with relapsed or refractory lymphoproliferative diseases. Clin. Cancer Res. 20, 5641-5651 (2014).

45. Wang, $K$. et al. LXRalpha promotes cell metastasis by regulating the NLRP3 inflammasome in renal cell carcinoma. Cell Death Dis. 10, 159 (2019). 